\title{
Sports Psychological Measurement And Statistical Method from the Perspective of Sports Public Service
}

\author{
Xiaopeng Liu $^{1}$ \\ ${ }^{1}$ Department of Physical Education, Dali University, Dali Yunan, 671000, China
}

\author{
Keywords: Square Dance; Sports Public Service; Rural Sports; Villager
}

\begin{abstract}
In this paper, the literature and data induction method is used to study the rural square dance from the perspective of the supply mechanism of sports public service. The study found that problems such as single supply subject, limited scope of supply and inadequate supply power still existed in the rural square dance. Secondly, in view of the above problems, this paper adopts the measures to diversify the supply mechanism, expand the scope of supply and improve the supply power. Finally, this paper puts forward the prospect and two suggestions for the future development of rural square dance.
\end{abstract}

\section{Introduction}

Sports public service is the general term for providing sports public goods and services, including the strengthening of sports public facilities, the development of sports public utilities, the release of sports public information, which will provide social security and create conditions for enriching the public life and participating in social sports activities [1]. The construction of sports public service plays an important role in the development of rural square dance, and it is also the inevitable demand for the development of rural sports. As a popular sports item in rural areas, square dance is popular among rural areas and gets rapid development. The characteristics of recreational sports of square dance has been deeply loved by the rural villagers, together with the implementation of the national fitness program and the local government's wide attention, it promotes the spread of square dance in rural areas to a certain extent. However, in the period of social transformation, the development of China's sports public services has shown a clear non-equalization situation, which has actually caused the inequality of our citizens' sports rights [2]. In terms of the development of rural square dance, there is a clear difference between the rural areas and the urban areas regardless of origin implementation, exercise conditions, activities carried out, which highlights the uneven development of sports public services in a sense. Therefore, through the study of rural square dance, this paper provides some suggestions for optimizing the sports public services in rural sports from the supply mechanism of the sports public service.

\section{Problems of Sports Public Service}

Contemporary China is in the period of social transformation, the objective existence of economic development and interest differentiation, social progress and contradiction, institutional reform and the lag of laws, concept change and moral anomie determines the great importance of building a harmonious society and constructing a public service system [2]. However, as an important part of public service, sports play an important role in comprehensively promoting the national construction plan. At present, the square dance is booming in the countryside, but there is still a serious lag compared with the city. On the basis of consulting the relevant literature, it is found that the study of rural square dance is only carried out separately, and it is not explored from other perspectives. Therefore, the research perspective is relatively narrow, there are obvious shortcomings. This paper which explores the problems of rural square dance from the supply mechanism of sports public service is still relatively rare.

At present, the supply of sports public services to the rural square dance mainly comes from the villagers self-financing, plus the village committee or funds provided by some individuals with 
economic strength. Of course, this approach is limited to some affluent villages, in other relatively backward areas, and most of the villagers organize it spontaneously. However, the villagers are greatly influenced by many factors such as time, space and facilities, and the existence of the above factors will greatly prevent villagers from organizing to carry out rural square dance. Therefore, the instability of the self-organized form of exercise and the long-term lack of supply subject ware bound to bring difficulties for the rapid development of rural square dance. In addition, as the supply subject of sports public service, the exercise mode organized by villagers spontaneously is too single obviously compared with the stable sports public service supply mechanism formed by the city square dance, therefore, it is inevitable for the rural square dance to fall behind the city square dance.

The single supply subject will affect the scope of supply of rural square dance to a certain extent. Venues, facilities and audio equipment of square dance are purchased by villagers, but the villagers with limited economic capacity can only buy some simple equipment. The survey found that a large part of equipment for square dance is purchased by individuals with economic strength due to the weak organization of villagers, and the equipment purchased is relatively poor. However, there are less other public institutions to buy rural sports public services, and the supply involving the venue, the square dance competition organization is relatively rare. The rural square dance as part of the sports public service, due to the private purchase of sports public service, if people are absent from the square dance exercise for personal factors (sports public service buyers), it is bound to affect the development of rural square dance.

At present, rural sports can't match with that of the city in terms of population density, sports public service facilities, spending power or sports market. Then, the shortage of rural sports public service will become inevitable. In this way, it is not surprising for villagers to organize square dance exercise spontaneously. However, the single supply subject and the limited supply scope is the embodiment of inadequate supply power to a certain degree, because the supply power mainly comes from the value of the existence of the sports market. However, due to the wide distribution of rural population, lack of rural spending power and the lack of sports market value or non-developed market value ultimately led to the lack of supply mechanism in terms of rural sports public service.

\section{Psychological Measurement}

Sports measurement and evaluation are both linked and differential, and the relationship between the two is very close. Physical measurement gives correct performance from number on various attributes or characteristics of human body in sports activities by instrument or other means. Specifically, the main task of measurement is to provide quantitative data for body shape, physical fitness, and technical aspects by means of certain measures. Although there is also the nature of the analysis, it not includes the explanation of it meaning in accordance with value. It can be seen that visible measurement describes the date from the perspective of number, which belongs to practical judgment, it provides quantitative information for the evaluation, but it is not that evaluation. Sports evaluation refers to the value judgment of sports phenomenon in accordance with certain value standards. In other words, it explains the phenomenon of sports from the perspective of value. This is to say that sports evaluation is related to the concept of sports goals and values, and it measures the development changes of students and the practice results of teaching planning according to sports goals and sports values. The focus of the evaluation is to determine the value of the sport as a standard. However, to make value judgments, it must be based on reliable information. The information here refers to two types of information, that is, quantitative and non-quantitative information. The former is obtained by measuring at a certain scale, while the latter is obtained by observing records and other means. In 1971, American scholar Gronlund argued that the evaluation could be simply expressed as follows: evaluation = measurement (quantity description) or non-measurement (quality account) + value judgment, that is to say, the evaluation is the activity of value judgments based on the quantity or quality. Grange's this formula is rough, but it did catch the essence of the evaluation activities. It can be seen that the evaluation is inseparable from the measurement, the task of measurement is the provide information from the number in the process of 
evaluation. Quantitative information plays an important role in the current sports evaluation, and must be given due attention. However, on the other hand, quantitative information also has some limitations in the evaluation. This is because some evaluation factors are difficult to quantify in the current sports learning, which needs to be further researched, and the judgment must also respect and use the above two types of information. From the purpose, the purpose of measurement and evaluation is not the same. The measurement is concerned with the quantitative effect of physical education, and the ultimate goal of evaluation is to maximize the role of sports goals and values, which is the work related to the purpose of sports and values. The evaluation process determines the development changes of students based on the goal of physical education, the implementation of physical education and teaching results and value process. It will produce different sports goals if see different ideas and values from this point of view, and the focus of evaluation function will also be different.

\section{Conclusion}

The problems of rural square dance have reflected the shortcomings of sports supply mechanism to a certain extent. The government's single supply subject, the limitation of supply scope and the lack of supply power have greatly influenced the development of rural square dance. However, in terms of the above problems, this paper has taken strong measures to solve the problem of rural square dance. However, the measures are bound to lag behind to a certain degree with the continuous development of society. Therefore, in order to better promote the future development of rural square dance, this paper puts forward the following suggestions from the supply mechanism of sports public services: (1) the establishment of rural square dance supervision mechanism. As the rural square dance is organized and formed by villagers spontaneously, some factors still exist, such as weak organization, low exercise enthusiasm and so on. Therefore, it is necessary to establish a government-led rural square dance regulatory pattern with villagers participating in. (2) the implementation of equal sports public services. The problem of equalization of public services is related to the national happiness index of a nation, but also reflects the level of economic development and the difference between rich and poor [4]. As a result, the government should vigorously support the construction of rural sports public services, and give a certain tilt in policy. Try to achieve balanced development between the urban square dance and rural square dance, and should take appropriate support in terms of funds, venues and facilities of rural square dance.

\section{References}

[1] Zhang, Z., Ou, J., Li, D., \& Zhang, S. (2017). Optimization Design of Coupling Beam Metal Damper in Shear Wall Structures. Applied Sciences, 7(2), 137.

[2] Shen, W., Li, D., Zhang, S., \& Ou, J. (2017). Analysis of wave motion in one-dimensional structures through fast-Fourier-transform-based wavelet finite element method. Journal of Sound and Vibration, 400, 369-386.

[3] Weisen Pan, Shizhan Chen, Zhiyong Feng. Automatic Clustering of Social Tag using Community Detection. Applied Mathematics \& Information Sciences, 2013, 7(2): 675-681.

[4] Cui Liu, Yanhu Li, Yingyue Zhang, Chuluo Yang, Hongbin Wu, Jingui Qin, and Yong Cao, Solution-Processed, Undoped, Deep-Blue Organic Light-Emitting Diodes Based on Starburst Oligofluorenes with a Planar Triphenylamine Core, Chemistry - A European Journal, 2012, 18(22), 6928-6934.

[5] Dalia Abdelhamid, Hulya Arslan, Yingyue Zhang, and Kathryn E. Uhrich, Role of Branching of Hydrophilic Domain on Physicochemical Properties of Amphiphilic Macromolecules, Polymer Chemistry, 2014, 5(4), 1457-1462. 